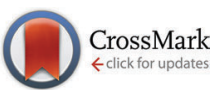

Cite this: Phys. Chem. Chem. Phys., 2015, 17, 28186

Received 16th January 2015 , Accepted 17th March 2015

DOI: $10.1039 / c 5 c p 00280 j$

www.rsc.org/pccp

\title{
Influence of Sn content on the hydrogenation of crotonaldehyde catalysed by colloidally prepared PtSn nanoparticles $\dagger$
}

\author{
Lena Altmann, ${ }^{a}$ Xiaodong Wang, ${ }^{\mathrm{b}}$ Holger Borchert, ${ }^{\mathrm{c}}$ Joanna Kolny-Olesiak, ${ }^{\mathrm{c}}$ \\ Volkmar Zielasek, ${ }^{a}$ Jürgen Parisi, ${ }^{\mathrm{c}}$ Sebastian Kunz ${ }^{* a}$ and Marcus Bäumer ${ }^{\mathrm{a}}$
}

\begin{abstract}
Bimetallic PtSn nanoparticles (NPs) of well-defined size and metal composition were prepared by means of colloidal methods. The mean particle diameter was about $2 \mathrm{~nm}$ for all samples irrespective of the $\mathrm{Pt} / \mathrm{Sn}$-ratio, which enables a systematic study of the influence of the composition on the catalytic properties while excluding particle size effects. The hydrogenation of crotonaldehyde was investigated as a reaction for which chemoselectivity is known to be a challenging task. Already very low atomic Sn contents $(\approx 10 \%)$ were found to lead to a significantly improved activity which may be attributed to an electronic effect of Sn on Pt. For further increasing tin contents the activity decreased gradually. This trend was accompanied by a steady increase in selectivity towards the desired product (crotylalcohol). The results show that the highest crotylalcohol time yields can be obtained by using catalysts with an atomic Sn content of approximately $23 \%$. In contrast, maximum crotylalcohol selectivities are achieved by using catalysts with a high tin content (>50\%).
\end{abstract}

\section{Introduction}

Unsaturated alcohols such as crotylalcohol, which can be obtained by selective hydrogenation of the corresponding $\alpha, \beta$-unsaturated aldehyde, are valuable building blocks for many fine chemicals such as pharmaceuticals and flavors. ${ }^{1}$ However, due to the presence of two different types of unsaturated chemical bonds $(\mathrm{C}=\mathrm{C}, \mathrm{C}=\mathrm{O})$ which can be hydrogenated, the selective hydrogenation of crotonaldehyde to crotylalcohol is a challenging task. The possible reaction pathways are illustrated in Scheme 1. While hydrogenation of the carbonyl group yields the desired product (crotylalcohol), hydrogenation of the $\mathrm{C}=\mathrm{C}$-bond leads to the formation of the undesired saturated aldehyde (butanal). Further side reactions that reduce the yield of the desired product are isomerization of crotylalcohol to butanal and decarbonylation of crotonaldehyde leading to the formation of propene and $\mathrm{CO}^{2}$

For monometallic late transition metal catalysts such as Pt, $\mathrm{Rh}, \mathrm{Pd}$ the selectivity towards the unsaturated alcohol is often

\footnotetext{
${ }^{a}$ Institute of Applied and Physical Chemistry and Center for Environmental Research and Sustainable Technology, University of Bremen Leobener Str. UFT, D-28359 Bremen, Germany. E-mail: SebKunz@Uni-Bremen.de

${ }^{b}$ Technical Electrochemistry, Faculty of Chemistry Technische Universität München, Lichtenbergstrasse 4, D-85748 Garching, Germany

${ }^{c}$ Energy and Semiconductor Research Laboratory, Department of Physics, University of Oldenburg, Carl-von-Ossietzky-Str. 9-11, D-26129 Oldenburg, Germany $\dagger$ Electronic supplementary information (ESI) available. See DOI: 10.1039/ c5cp00280j
}

very low and the saturated aldehyde is obtained as the main product. ${ }^{3}$ The high selectivity towards the undesired $\mathrm{C}=\mathrm{C}$-bond hydrogenation is attributed to the fact that hydrogenation of the

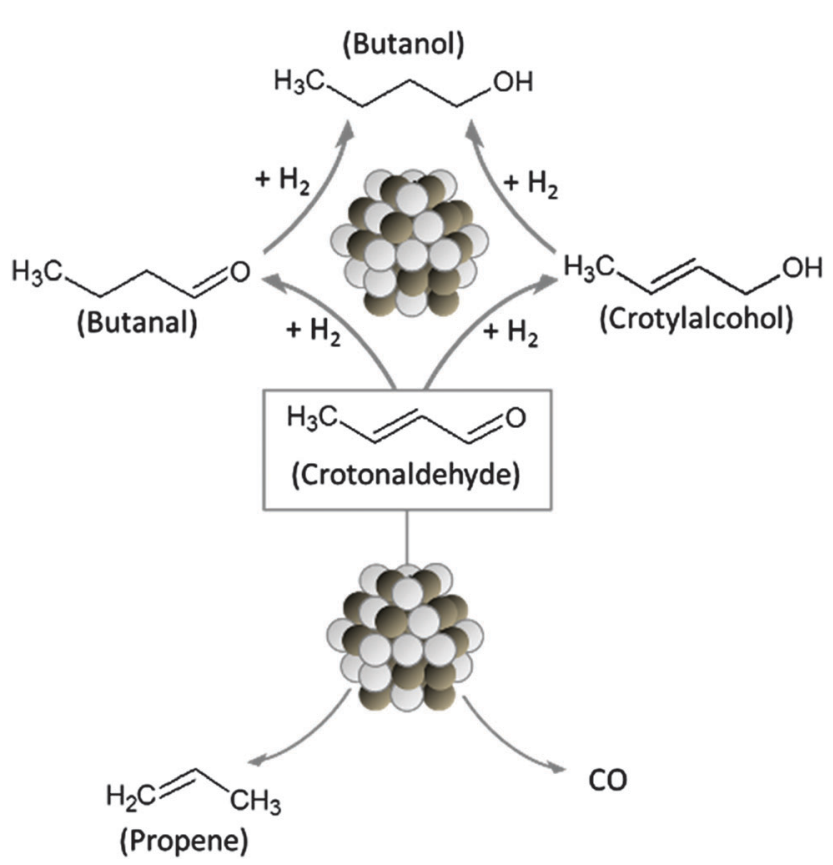

Scheme 1 Overview over the possible reaction pathways for the hydrogenation of crotonaldehyde catalyzed by PtSn nanoparticles. 
$\mathrm{C}=\mathrm{C}$ bond is thermodynamically favoured in comparison to hydrogenation of the $\mathrm{C}=\mathrm{O}$ group. ${ }^{4}$ Furthermore, the interaction of $\mathrm{C}=\mathrm{O}$-groups with pure noble metals is usually weak compared to $\mathrm{C}=\mathrm{C}$-groups, which makes hydrogenation of the latter more likely. ${ }^{5}$ Several parameters have been discussed to affect the selectivity such as particle size, ${ }^{2}$ strong metal support interactions (SMSI), ${ }^{6}$ metal composition ${ }^{1}$ or additives like alkyl bromides. ${ }^{7}$ While the maximum crotylalcohol selectivity reported for gas phase hydrogenation of crotonaldehyde with monometallic Pt catalysts (without SMSI effects) was around $40 \%,{ }^{1,2}$ the selectivity was shown to be enhanced up to $77 \%$ by addition of a second metal such as Sn. ${ }^{8}$ The origin of the selectivity improvement for bimetallic catalysts has been discussed in terms of alloy formation as well as $\mathrm{SnO}_{x}$ patches, decorating the noble metal particle surface, both creating new adsorption sites at the interface. ${ }^{3,9,10}$ $\mathrm{SnO}_{x}$ patches at the particle surface are suggested to act as Lewis acid sites which favour coordination of the $\mathrm{C}=\mathrm{O}$ bond to the catalyst surface via the lone pair electrons of oxygen, ${ }^{11}$ thus enhancing its reactivity. ${ }^{1}$ Likewise, a charge transfer from $\mathrm{Sn}$ to Pt was proposed for PtSn alloys, ${ }^{12}$ leading to positively polarized Sn which acts as Lewis acid site thus favouring the coordination of the $\mathrm{C}=\mathrm{O}$ bond in analogy to $\mathrm{SnO}_{x}{ }^{1}$ Besides the formation of new adsorption sites at the surface by $\mathrm{Sn}$ and $\mathrm{SnO}_{x}$, an ensemble effect for Sn on Pt was also discussed leading to a dilution of adjacent $\mathrm{Pt}$ surface atoms that are capable to activate $\mathrm{C}=\mathrm{C}$ bonds. ${ }^{13}$ Whether metallic $\mathrm{Sn}$ or $\mathrm{SnO}_{x}$ is the more relevant species for an effective selectivity improvement is, however, not unequivocally clarified at this point. ${ }^{9,10,14}$

Even though bimetallic catalysts and their chemoselectivity in the hydrogenation of $\alpha, \beta$-unsaturated aldehydes have been intensively studied in the past, systematic studies regarding the effect of the tin content on the selectivity are scarce and results are contradictory. ${ }^{1,13-15}$ Some authors reported a certain trend for the dependency of activity and selectivity on the catalyst composition, ${ }^{8,13}$ whereas other authors observed significant deviations from a linear dependency of catalytic properties on the catalyst composition. ${ }^{14,16}$ These discrepancies may be attributed to the difficulty to prepare nanoparticles (NPs) of well-defined size and chemical composition by conventional catalyst preparation methods. Such methods require the presence of the support material during particle synthesis which however affects the reducibility and nucleation of the metals. As a result, nanoparticle size and composition are strongly influenced by the support. ${ }^{11,13,17}$ For PtSn catalysts, prepared by conventional techniques that require the presence of a support material, it has been shown that significant amounts of Sn decorate the support surface in a highly dispersed state rather than being incorporated into the particles. ${ }^{18,19}$ As a consequence, the actual tin content of the bimetallic particles can significantly vary from particle to particle and differ from the overall tin content of the catalyst. These drawbacks of conventional preparation techniques can be overcome by colloidal synthesis. Due to the high control over metal composition and particle size as well as the possibility to deposit the particles on various supports after their preparation, these methods hence exhibit high potential for systematic investigations in heterogeneous catalysis. ${ }^{17,20,21}$
Herein we present a systematic study regarding the influence of the tin content on the chemoselective hydrogenation properties of bimetallic PtSn nanoparticles (NPs). The applied colloidal preparation method enables to adjust the Sn content of the bimetallic particles over a wide range of compositions, while keeping the particle size constant. ${ }^{20}$ Similar to other colloidal preparation methods, the synthesis route used in this study requires the presence of organic ligands during synthesis to stabilize the nanoparticles against agglomeration. However, it has been shown previously for monometallic Pt NPs that under steady state conditions the protecting ligands dodecylamine (DDA) do neither affect the activity nor the selectivity in the hydrogenation of crotonaldehyde. ${ }^{22}$ This finding was related to spillover of the DDA ligands from the particle surface to the support, as the ligands have to compete with the reactant and the reaction intermediates (such as crotonaldehyde and $\mathrm{CO}$ ) for the adsorption sites on the particles. ${ }^{22}$ In this way, an influence of the protecting ligands and the particle size on the catalytic properties of the colloidally prepared PtSn NPs can be excluded and changes in the selectivity of the metal nanoparticles can be related exclusively to changes in the metal composition.

\section{Experimental section}

\subsection{Preparation of monometallic DDA-functionalized Pt nanoparticles}

An alkaline ethylene glycol (EG, Sigma Aldrich; $\geq 99.5 \%$ ) method was used to prepare monometallic Pt nanoparticles. ${ }^{23} 5 \mathrm{ml}$ of an EG solution containing $\mathrm{H}_{2} \mathrm{PtCl}_{6} \cdot 6 \mathrm{H}_{2} \mathrm{O}(100 \mathrm{mg} ; 41 \mathrm{mM})$ (Chempur; $37.5 \mathrm{wt} \% \mathrm{Pt}$ basis) were added to an equal amount of an alkaline $\mathrm{NaOH}-\mathrm{EG}$ solution (0.4 M) ( $\mathrm{NaOH}$; Fluka; >97\%). After 15 min of stirring at ambient temperature the yellow solution was heated to $160{ }^{\circ} \mathrm{C}$ using an oil bath. At an oil bath temperature of approximately $120{ }^{\circ} \mathrm{C}$ the mixture turned black indicating the formation of colloidal Pt NPs. The reaction was kept at $160{ }^{\circ} \mathrm{C}$ under vigorous stirring for $3 \mathrm{~h}$ to ensure quantitative reduction and was then allowed to cool down to room temperature. The as-prepared "unprotected" Pt NPs were functionalized in a separate step via a phase-transfer reaction. Therefore, the EG solution of "unprotected" Pt NPs was diluted with EG to obtain a Pt concentration of $10.2 \mathrm{mM}$. The diluted NP solution was then added dropwise into an equal volume of a dodecylamine (16.4 mM) (DDA; Merck; 95\%) containing toluene solution and the resulting mixture was vigorously stirred at ambient temperature for $5 \mathrm{~h}$. Two distinct phases were formed after $1 \mathrm{~h}$ of resting. The black color of the upper toluene phase and a clear EG phase confirmed successful phase transfer of the NPs. The EG phase was removed and the toluene phase was washed three times with deionized $\mathrm{H}_{2} \mathrm{O}$.

\subsection{Preparation of bimetallic DDA-functionalized PtSn nanoparticles}

DDA-functionalized bimetallic PtSn nanoparticles were prepared in a toluene-EG dual phase system. A detailed study on the synthesis of colloidal PtSn nanoparticles, their characterization 
and how the particle size, composition and shape can be tuned has been reported previously. ${ }^{20,24,25}$ For the synthesis of bimetallic nanoparticles with a nominal molar metal ratio of $1: 1,5.7 \mathrm{mg}$ $\mathrm{SnCl}_{2} \cdot 2 \mathrm{H}_{2} \mathrm{O}$ (Sigma-Aldrich, 98\%) was dissolved in $1.5 \mathrm{ml}$ EG (17 mM). This solution was added to $2.5 \mathrm{ml}$ of a toluene solution containing $\mathrm{PtCl}_{4}(8.5 \mathrm{mg} ; 10 \mathrm{mM}$ ) (Sigma-Aldrich, 99.9\%) and DDA (280 mg; 0.6 M) (Sigma-Aldrich, 98\%) at room temperature under vigorous stirring. $1 \mathrm{ml}$ of a freshly prepared toluene solution containing didodecyldimethylammonium bromide (DDAB; $47.2 \mathrm{mg} ; 0.104 \mathrm{M}$ ) (Sigma-Aldrich, 98\%) and tetrabutylammonium borohydride (TBAB; $50 \mathrm{mg} ; 0.155 \mathrm{M}$ ) (Sigma-Aldrich 98\%) was added to the toluene-EG dual phase system under vigorous stirring and the reaction mixture was heated immediately to $100{ }^{\circ} \mathrm{C}$ under an inert $\mathrm{N}_{2}$ atmosphere. For complete reduction of the metal salts the reaction mixture was kept at $100{ }^{\circ} \mathrm{C}$ for $1 \mathrm{~h}$ and then allowed to cool down to room temperature. After $1 \mathrm{~h}$ of resting, the upper dark brown toluene phase containing the bimetallic nanoparticles was separated from the clear EG solution.

Nanoparticles with nominal Pt:Sn metal ratios of $5: 1,3: 1$ and $1: 3$ were prepared using the same procedure and adjusting the relative amounts of $\mathrm{Sn}$ and Pt precursor. For the catalysts with a metal ratio of $1: 1,3: 1$ and $5: 1$ the amount of $\mathrm{PtCl}_{4}$ used was the same $\left(8.5 \mathrm{mg}\right.$ ) and the amount of $\mathrm{SnCl}_{2} \cdot 2 \mathrm{H}_{2} \mathrm{O}$ was adjusted corresponding to the desired ratio. The DDA-PtSn sample with a $\mathrm{Pt}: \mathrm{Sn}$ metal ratio of $1: 3$ was prepared using $4.4 \mathrm{mg} \mathrm{PtCl}_{4}$ and $8.7 \mathrm{mg} \mathrm{SnCl}_{2} \cdot 2 \mathrm{H}_{2} \mathrm{O}$.

\subsection{Deposition of the nanoparticles on the $\mathrm{TiO}_{2}$ support}

The mono- and bimetallic DDA-functionalized nanoparticles were precipitated by adding a threefold volume of methanol to the colloidal toluene dispersion and then isolated from the supernatant solution using centrifugation. The particle precipitate was redispersed in chloroform (Sigma Aldrich; 99\%) and $\mathrm{TiO}_{2}$ (Aldrich; 99.8\%) was added to obtain supported catalysts with a nominal loading of $1 \mathrm{wt} \%$ of Pt. The suspension was manually stirred with a spatula until evaporation of the solvent was completed. The obtained catalysts were dried in vacuum overnight, before they were characterized and tested for the hydrogenation of crotonaldehyde. From various tested metaloxide supports the applied $\mathrm{TiO}_{2}$ was the only material that did not cause any significant background reactions of crotonaldehyde and was therefore chosen for the present study.

\subsection{Determination of the particle size}

TEM (Transmission Electron Microscopy) and EDX (EnergyDispersive X-ray Spectroscopy) analysis was performed using a Tecnai F20 S-TWIN microscope (FEI), equipped with an EDX detector. TEM images were taken at an acceleration voltage of $200 \mathrm{kV}$ and a magnification of $185 \mathrm{k}$.

Samples for TEM/EDX measurements were prepared by placing a drop of the supported NP catalysts, previously dispersed in ethanol, on a carbon-coated copper grid (Quantifoil ${ }^{\circledR}$, $\mathrm{Cu} 200 \mathrm{mesh}$ ). ImageJ (open source software) was used to determine the average particle diameter and the corresponding standard deviation from TEM images for every sample by counting approximately 250 particles.

\subsection{Determination of the metal loading}

A Perkin Elmer DV 7300 was applied to determine the metal composition by inductively coupled plasma (ICP) analysis. The metals were dissolved by boiling the catalyst powders in aqua regia for $1 \mathrm{~min}$ and digesting them further at room temperature overnight.

\subsection{Crotonaldehyde hydrogenation}

The gas-phase hydrogenation of crotonaldehyde (Merck; 99\%) was investigated at atmospheric pressure in a quartz tube reactor with an inner diameter of $4 \mathrm{~mm}$. Differential conditions were achieved by keeping the conversion level of crotonaldehyde below $12 \%$. In a typical experiment, 5-70 $\mathrm{mg}$ of the catalyst (grain size of 100-280 $\mu \mathrm{m}$ ) were diluted with $354 \mathrm{mg}$ quartz sand (Roth; $>99 \%$ ) of the same grain size in order to ensure plug flow hydrodynamics and to prevent heat transport limitations. The diluted catalyst was placed between glass wool in the quartz reactor yielding a catalyst bed height of about $2 \mathrm{~cm}$. The gas flow rate was set to $50 \mathrm{~cm}^{3} \mathrm{~min}^{-1}$, containing 1 vol\% of crotonaldehyde, 20 vol\% of hydrogen and $79 \% \mathrm{~N}_{2}$ as inert diluent (all gases were purchased from Lindegas with a purity grade of 5.0.). Isothermal reaction conditions with a reactor temperature of $80{ }^{\circ} \mathrm{C}$ were controlled using a thermocouple connected to a PMA KS-40 control unit. The gas stream at the reactor outlet was analyzed using an Agilent 7890A gas chromatograph equipped with a flame ionization detector and a CS FSINNOPEG-1000 capillary column (50 m, ID $0.25 \mathrm{~mm}$, film thickness $0.1 \mu \mathrm{m}$ ). Helium (Lindegas; 5.0) was used as carrier gas and the gas velocity was set to $40 \mathrm{~cm}^{3} \mathrm{~s}^{-1}$. In order to achieve separation of the different components, the temperature of the GC oven was held at $65{ }^{\circ} \mathrm{C}$ for $5.5 \mathrm{~min}$ and subsequently heated to $200{ }^{\circ} \mathrm{C}$ at a rate of $120{ }^{\circ} \mathrm{C} \min ^{-1}$. Every $15 \mathrm{~min}$ the gas composition at the reactor outlet was analyzed by using a gas sampling valve with a $6 \mu \mathrm{l}$ sample loop until steady state conditions of the reaction were reached after about $120 \mathrm{~min}$. The reaction rate $\left(\mathrm{mol} \mathrm{s}^{-1} \mathrm{~g}_{(\mathrm{Pt}+\mathrm{Sn})}{ }^{-1}\right)$ was calculated from the total amount of products and the selectivity is given by the relative amount of the respective product. The turn over frequency (TOF) was determined by normalizing reaction rate $\left(\mathrm{mol} \mathrm{s}^{-1} \mathrm{~g}_{(\mathrm{Pt}+\mathrm{Sn})}{ }^{-1}\right)$ to the number of surface atoms. The number of surface atoms was calculated from the mean particle diameter determined by TEM and the total metal loading of the catalysts measured by ICP, while assuming the same covalent radius for both metals $(d=0.136)$ for reasons of simplification, a packing density of $74 \%$, and a spherical shape. The experimental error of TOF and selectivity was determined from the standard deviation obtained for the $\mathrm{PtSn} / \mathrm{TiO}_{2}$ catalyst with 55 at\% $\mathrm{Sn}$ by repeating the measurement 3 times with the same (fresh) sample.

\section{Results and discussion}

\subsection{Catalyst characterization}

Mono- and bimetallic nanoparticles with different tin contents were prepared following a colloidal synthesis route described recently, which allows for preparation of bimetallic PtSn nanoparticles well-defined in size and composition. ${ }^{20}$ Particle size 
and metal composition of the obtained nanoparticles were determined by TEM and EDX analysis (see Table 1). The catalyst samples are denoted as "Pt/TiO ${ }_{2}$ " or " $\mathrm{PtSn}(x) / \mathrm{TiO}_{2}$ ", respectively, with $x$ corresponding to the atomic Sn content in \% relative to the total number of metal atoms $(\mathrm{Sn}+\mathrm{Pt})$. The particles exhibit similar mean diameters of about $2 \mathrm{~nm}$ and narrow size distributions irrespective of the tin content. In agreement with our previous findings the actual tin content of all samples determined by EDX was found to be lower than the nominal tin content calculated from the amount of precursor applied during synthesis. ${ }^{20}$ This indicates that $\mathrm{Sn}$ cannot be reduced quantitatively and demonstrates the relevance of characterizing the chemical composition of colloidal bimetallic nanoparticles.

After successful preparation the colloidal nanoparticles were deposited on $\mathrm{TiO}_{2}$ by impregnation yielding a homogeneous distribution of the nanoparticles over the support material as seen in Fig. 1a, showing a representative TEM image of the as prepared $\mathrm{PtSn}(55) / \mathrm{TiO}_{2}$ catalyst (see ESI, $\dagger$ Fig. S1 for all other catalysts).

\subsection{Catalysis experiments}

The well-defined bimetallic nanoparticles were tested with respect to their catalytic properties for the hydrogenation of crotonaldehyde. However, no clear dependence for the activity and selectivity on the Sn content was observed (Fig. 2a-c). Compared to the crotylalcohol selectivities reported in literature for PtSn of up to $77 \%,{ }^{8}$ the as-prepared catalyst showed comparatively low crotylalcohol selectivities with a maximum value of $S_{\text {crotyl }}=34 \%$ for the catalyst containing $30 \%$ Sn. We have shown previously for monometallic Pt NPs that DDA does not affect the activity and selectivity in the hydrogenation of crotonaldehyde under state conditions. ${ }^{22}$ Therefore, it is unlikely that DDA is the reason for this low selectivity and the nonlinear activity and selectivity dependence with respect to the actual Sn content. However, a Sn surface enrichment and the presence of $\mathrm{SnO}_{x}$ were observed in a recent XPS investigation of the as-prepared nanoparticles synthesized by the recipe applied in the present study. ${ }^{25}$ In order to check whether a reduction of $\mathrm{SnO}_{x}$ to metallic $\mathrm{Sn}$ is beneficial for a selectivity improvement, the catalysts were reduced in situ at $230{ }^{\circ} \mathrm{C}$ in $10 \% \mathrm{H}_{2} / 90 \% \mathrm{~N}_{2}$ (flow rate $50 \mathrm{~cm}^{3} \mathrm{~min}^{-1}$ ) for $1 \mathrm{~h}$ and subsequently applied as hydrogenation catalysts for crotonaldehyde.

After the reductive pre-treatment significant activity and selectivity improvements and a clear trend for the selectivity

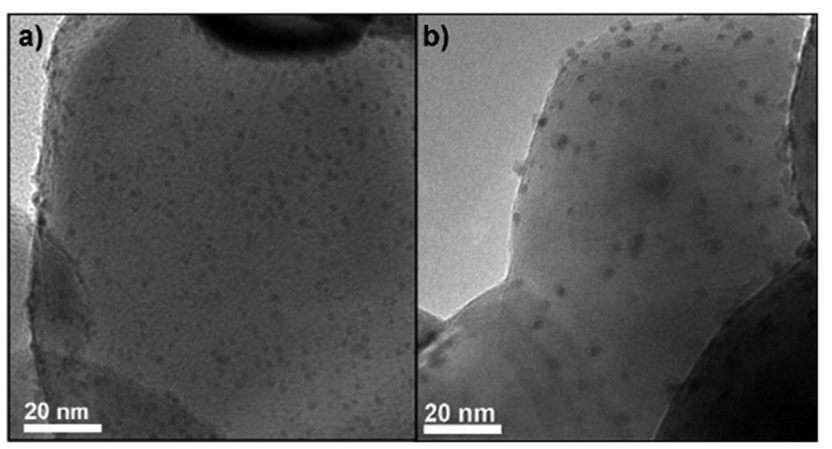

Fig. 1 TEM images of $\mathrm{PtSn}(55) / \mathrm{TiO}_{2}$ (a) before catalysis and (b) after catalysis with pre-reduction in $10 \% \mathrm{H}_{2}$ (rest $\mathrm{N}_{2}$ ) at $230{ }^{\circ} \mathrm{C}$ for $1 \mathrm{~h}$.

with respect to the tin content were observed (Fig. 2d and e). In agreement with a previous study the selectivity towards crotylalcohol $\left(S_{\text {crotyl }}\right)$ is very low $(4 \%)$ for monometallic Pt. ${ }^{22}$ The TOF value of $4.9 \times 10^{-2} \mathrm{~s}^{-1}$ with respect to the conversion of crotonaldehyde is comparable to the value reported previously by another group for colloidally prepared Pt NPs of similar size and studied under similar conditions $(2.1 \times$ $10^{-2} \mathrm{~s}^{-1}$ ), but supported on SBA15. ${ }^{2}$ Compared to the monometallic Pt catalyst a pronounced enhancement of activity by almost one order of magnitude was achieved in the present study by addition of only $10 \% \mathrm{Sn}$. With further increasing Sn content the activity gradually declined. Whereas the activity showed a maximum at $10 \%$ tin and decreased at higher concentrations (see Fig. 2d), the selectivity towards crotylalcohol increased monotonically as the tin content was increased (Fig. 2e). TEM analysis after the reductive pre-treatment revealed a slight increase in particle size but the mean diameter was still within the same range for all catalysts (Table 1). As a consequence, particle size effects on the catalytic properties can be excluded. Likewise, an activity and selectivity enhancement resulting from metalsupport interactions induced by the low temperature reduction is unlikely, because activity and selectivity of the monometallic Pt catalyst were not affected by the reductive pre-treatment. As mentioned above, DDA ligands do not alter the catalytic properties of monometallic Pt NPs in the selective hydrogenation of crotonaldehyde under steady state conditions, but are proposed to diffuse from the particle onto the support. ${ }^{22} \mathrm{We}$ therefore conclude that the activity and selectivity enhancements after the reductive pre-treatment can be related to the reduction of $\mathrm{Sn}$, which indicates the necessity of metallic Sn for an effective improvement of the crotylalcohol selectivity.

Table 1 Structural parameter of the supported PtSn-catalysts

\begin{tabular}{|c|c|c|c|c|c|}
\hline Sample & $\begin{array}{l}\text { Pt loading } \\
\text { (wt } \%)\end{array}$ & $\begin{array}{l}\text { Sn content } \\
\text { (at } \%)\end{array}$ & $\begin{array}{l}\mathrm{Pt} / \mathrm{Sn} \text {-ratio } \\
\text { nominal }\end{array}$ & $\begin{array}{l}\text { Particle size }^{c} \\
\text { as-prepared (nm) }\end{array}$ & $\begin{array}{l}\text { Particle size }^{c} \\
\text { pre-reduced }(\mathrm{nm})\end{array}$ \\
\hline $\mathrm{Pt} / \mathrm{TiO}_{2}$ & 0.4 & 0 & - & $1.8 \pm 0.3$ & $2.1 \pm 0.3$ \\
\hline $\operatorname{PtSn}(10) / \mathrm{TiO}_{2}$ & 1.3 & 10 & $5: 1$ & $2.0 \pm 0.3$ & $2.6 \pm 0.3$ \\
\hline $\mathrm{PtSn}(23) / \mathrm{TiO}_{2}$ & 1.2 & 23 & $3: 1$ & $1.9 \pm 0.3$ & $2.1 \pm 0.3$ \\
\hline $\mathrm{PtSn}(30) / \mathrm{TiO}_{2}$ & 1.1 & 30 & $1: 1$ & $2.1 \pm 0.3$ & $2.6 \pm 0.3$ \\
\hline $\mathrm{PtSn}(55) / \mathrm{TiO}_{2}$ & 0.6 & 55 & $1: 3$ & $2.0 \pm 0.3$ & $2.2 \pm 0.3$ \\
\hline
\end{tabular}

${ }^{a}$ Determined from ICP-analysis. ${ }^{b}$ Determined from EDX-analysis. ${ }^{c}$ Determined from TEM-analysis. 

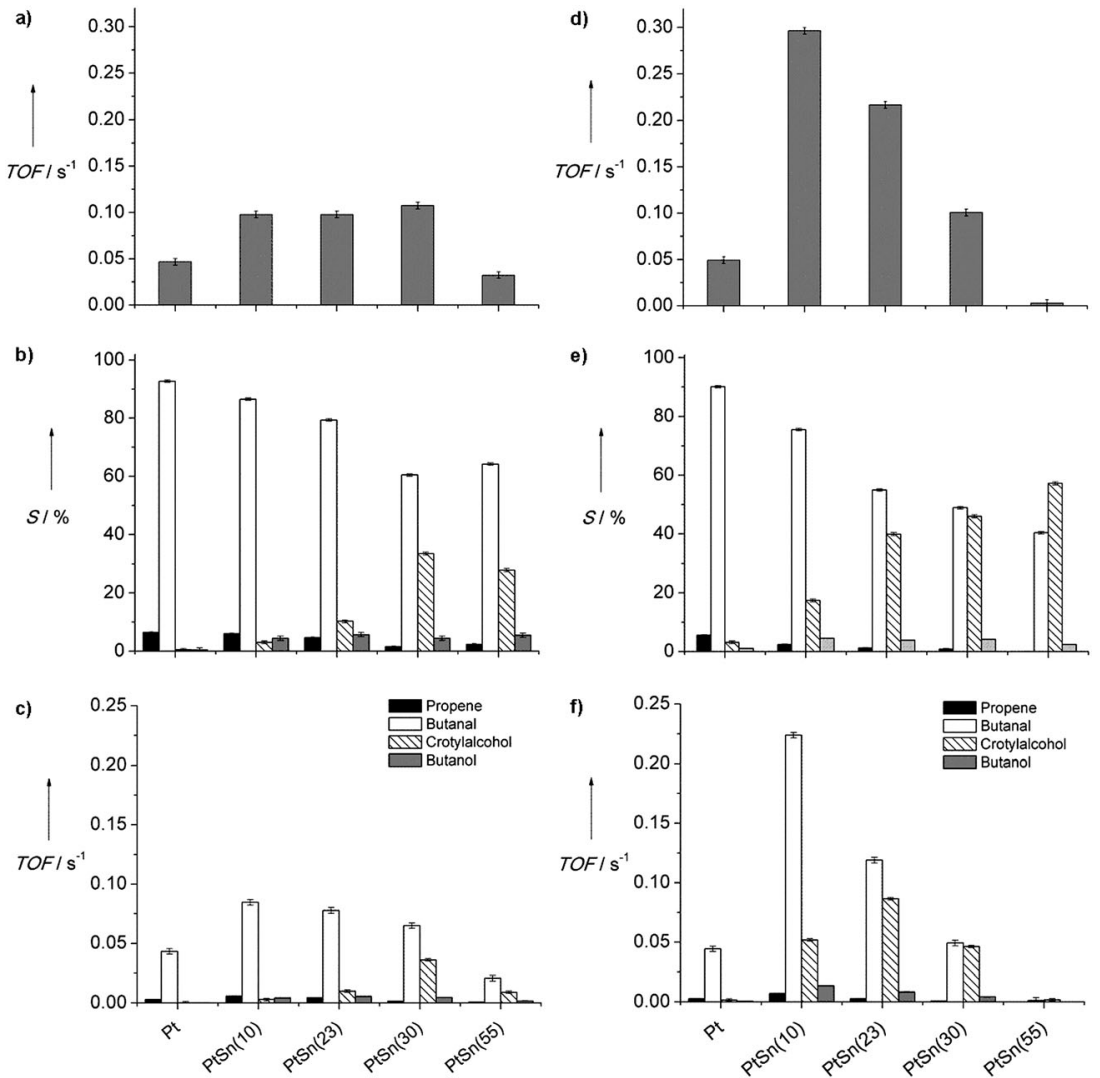

Fig. 2 TOF $\left(\mathrm{s}^{-1}\right)$ with respect to the conversion of crotonaldehyde, selectivity and TOF $\left(\mathrm{s}^{-1}\right)$ with respect to the formation of the individual products formed by the hydrogenation of crotonaldehyde as a function of Sn content for the as-prepared $(a-c)$ and the pre-reduced catalysts ( $d-f)$, respectively. The gas flow rate was set to $50 \mathrm{ml} \mathrm{min}{ }^{-1}$ containing 1 vol\% crotonaldehyde, 20 vol\% $\mathrm{H}_{2}$ and $\mathrm{N}_{2}$ as balance. All measurements were conducted under isothermal conditions at $80^{\circ} \mathrm{C}$.

Unfortunately, we cannot further supported this hypothesis by conventional post characterizations as tin readily oxidizes in the presence of any oxygen. Instead in situ XPS would be necessary, which was however not available for this project. Complete reduction of the tin within the particles cannot be assumed to occur in the present case (reduction at $230{ }^{\circ} \mathrm{C}$ ), because even at temperatures above $500{ }^{\circ} \mathrm{C}$ quantitative reduction of $\mathrm{Sn}$ in bimetallic $\mathrm{PtSn}$ catalysts was reported to be challenging. ${ }^{11,13,26}$ (To avoid sintering and SMSI effects, which exclude the possibility to assign changes in catalytic properties exclusively to the influence of the Sn content, the reduction temperature was not further increased than $230{ }^{\circ} \mathrm{C}$.)

The observed decrease of the catalyst activity as the Sn concentration was increased from $10 \%$ to $55 \%$ could be assigned to a dilution of Pt by tin. Rao et al. ${ }^{27}$ suggested that the surface reaction between adsorbed $\mathrm{H}$ and crotonaldehyde (as alkoxide surface intermediate) is the kinetically relevant step in the hydrogenation of crotonaldehyde. A decreasing reaction rate may therefore be attributed to a lower surface concentration of the two reactive intermediates. Different from the adsorption of crotonaldehyde, dissociative adsorption of $\mathrm{H}_{2}$ does occur merely on $\mathrm{Pt}$ but not on $\mathrm{Sn}$. We hence relate the Sn-induced decrease of activity above $10 \% \mathrm{Sn}$ to the loss of $\mathrm{Pt}$ surface sites that are prerequisite for the activation of $\mathrm{H}_{2}$. It has been previously shown that low amounts of $\mathrm{Sn}$ in combination with Pt lead to a pronounced promotion of the hydrogenation activity of Pt. As this effect has been assigned to an electronic modification of $\mathrm{Pt}$ by $\mathrm{Sn}$ we conclude the same effect for our sample with a tin concentration of $10 \% .^{28-30}$ The electronic modification leads to a lowering of the binding energies for atomic $\mathrm{H}$ and crotonaldehyde as reported in theoretical and experimental investigations. ${ }^{1,12}$ As a result the reactivity of the adsorbed surface intermediates can be assumed to increase. ${ }^{31}$

In contrast to the activity, the selectivity increases monotonically with increasing Sn content (Fig. 2e). In Fig. 2f, the TOF values with respect to the formation of the individual reaction products (i.e., the corresponding yield) are given as a function of $\mathrm{Sn}$ content. Both, the hydrogenation rate of $\mathrm{C}=\mathrm{C}$ (leading to the formation of butanal) and $\mathrm{C}=\mathrm{O}$ (leading to the formation of crotylalcohol), increase significantly upon addition of low $\mathrm{Sn}$ amounts. Interestingly, when the $\mathrm{Sn}$ content is further increased the hydrogenation rate of $\mathrm{C}=\mathrm{C}$ decreases together with the overall reaction rate, whereas the hydrogenation rate of the $\mathrm{C}=\mathrm{O}$ bond exhibits a maximum for a tin content of $23 \%$. This dependence of the $\mathrm{C}=\mathrm{O}$ bond hydrogenation rate on $\mathrm{Sn}$ may be related to the creation of new adsorption sites by $\mathrm{Sn}$ leading to an alteration of the most stable adsorption modes. ${ }^{12}$ Based on DFT calculations in combination with experimental studies, Haubrich et al. proposed that the most stable adsorption mode of crotonaldehyde on PtSn surfaces generally requires a 
$\mathrm{O}-\mathrm{Sn}$ bond leading to a preferential activation of the $\mathrm{C}=\mathrm{O}-$ bond. In contrast, additional adsorption modes containing di- $\sigma$ configuration of the $\mathrm{C}=\mathrm{C}$ bond are known for monometallic $\mathrm{Pt}$ that lead to the preferential hydrogenation of the $\mathrm{C}=\mathrm{C}$-bond. ${ }^{12,32}$ The improved activation of the $\mathrm{C}=\mathrm{O}$ bond by $\mathrm{O}-\mathrm{Sn}$ bond formation for $\mathrm{PtSn}(23)$ compared to the monometallic $\mathrm{Pt}$ or Pt-rich catalysts may thus account for the enhanced $\mathrm{C}=\mathrm{O}$ bond hydrogenation at decreasing overall hydrogenation rates. In relation to the hydrogenation of the $\mathrm{C}=\mathrm{O}$ bond, $\mathrm{C}=\mathrm{C}$ bond hydrogenation becomes less favourable due to the dilution of adsorption sites for both $\mathrm{C}=\mathrm{C}$ and $\mathrm{H}_{2}$. At tin contents higher than $23 \%$, the decreasing availability of $\mathrm{Pt}$ adsorption sites becomes more dominant and hence limits the $\mathrm{H}_{2}$ activation. As a consequence, the $\mathrm{C}=\mathrm{O}$-hydrogenation rates start to decrease, too. This demonstrates that for an optimization of both, activity and selectivity, the metal composition needs to be adjusted precisely. Such a precise adjustment of the metal composition requires a high control over structural parameters during catalyst preparation, which cannot be achieved by conventional catalysts preparation methods. In contrast, colloidal methods are suitable to address these challenges, emphasizing its value as model systems for catalysis studies. ${ }^{33}$

For the catalyst with the highest tin content used in the present study (55\%) a $S_{\text {crotyl }}$ value of $56 \%$ was achieved. This value lies within the broad range of values $(25 \% \text { to } 77 \%)^{8,11,14,34}$ previously reported by other groups for the gas phase hydrogenation of crotonaldehyde at atmospheric pressure and in absence of SMSI effects. The activity and selectivity dependencies with respect to the tin content observed in literature are however contradictory. Ruiz-Martínez et al. ${ }^{14}$ did not observe a monotonic trend for the selectivity, whereas an increasing selectivity with increasing Sn content of up to $75 \%$ was reported by Coloma et al. under comparable reaction conditions. ${ }^{13}$ As mentioned in the introduction, these discrepancies may be attributed to the difficulty to prepare nanoparticles well-defined in size and chemical composition using conventional catalyst preparation methods. For these methods, the particle formation is strongly influenced by the support material and differences in metal support interactions can lead to a different dispersion of the two metals on the support material. ${ }^{18,19}$ As a consequence, the actual tin content of the bimetallic particles can differ significantly from the overall tin content of the catalyst. In contrast, the colloidal method applied in this study enables to precisely control the tin content over a wide range of compositions while keeping the particle size constant. We can thus conclude that the selectivity for crotylalcohol over PtSn particles increases monotonically with increasing tin content as reported by Coloma et $a l^{13}$

\section{Conclusion}

Bimetallic PtSn NPs of different metal compositions but similar in size were prepared by means of colloidal methods and subsequently deposited on $\mathrm{TiO}_{2}$ for catalytic investigations. This enabled us to systematically study the influence of the composition on the catalytic properties for the hydrogenation of crotonaldehyde, while particle size effects could be excluded. The activity of Pt was found to increase significantly by adding small amounts of Sn (10\%), which can be related to an electronic effect. It however decreased gradually upon further increasing the tin content which can be explained by a loss of Pt surface sites required for the activation of $\mathrm{H}_{2}$. The decreasing activity was accompanied by a steady selectivity increase towards the desired product (crotylalcohol). While controversial observations and nonmonotonic dependencies can be found in literature concerning the influence of the metal composition, we could show that activity and selectivity follow clear trends with respect to the Sn content, which underlines the potential of colloidal chemistry for model studies in heterogeneous catalysis. Finally, the results demonstrate how by tuning the composition one can either achieve highest selectivities or optimize the crotylalcohol time yield for bimetallic PtSn nanoparticle catalysts.

\section{Acknowledgements}

We are grateful for financial support by Deutsche Forschungsgemeinschaft (DFG) (Project No. AL 431/11-1, BA 1710/15-1, BO 1973/5-1). S.K. acknowledges the "Fonds der Chemischen Industrie” (FCI) for financial support through a Liebig Habilitations Stipendium.

\section{References}

1 P. Claus, Top. Catal., 1998, 5, 51-62.

2 M. Grass, R. Rioux and G. Somorjai, Catal. Lett., 2009, 128, 1-8.

3 P. Mäki-Arvela, J. Hájek, T. Salmi and D. Y. Murzin, Appl. Catal., A, 2005, 292, 1-49.

4 M. A. Vannice and B. Sen, J. Catal., 1989, 115, 65-78.

5 F. Coloma, J. Llorca, N. Homs, P. R. de la Piscina, F. Rodriguez-Reinoso and A. Sepulveda-Escribano, Phys. Chem. Chem. Phys., 2000, 2, 3063-3069.

6 M. A. Vannice, Top. Catal., 1997, 4, 241-248.

7 E. Galloway, M. Armbrüster, K. Kovnir, M. S. Tikhov and R. M. Lambert, J. Catal., 2009, 261, 60-65.

8 J. L. Margitfalvi, G. Vankó, I. Borbáth, A. Tompos and A. Vértes, J. Catal., 2000, 190, 474-477.

9 K. Liberková and R. Touroude, J. Mol. Catal. A: Chem., 2002, 180, 221-230.

10 D. I. Jerdev, A. Olivas and B. E. Koel, J. Catal., 2002, 205, 278-288.

11 A. Huidobro, A. Sepúlveda-Escribano and F. RodríguezReinoso, J. Catal., 2002, 212, 94-103.

12 J. Haubrich, D. Loffreda, F. Delbecq, P. Sautet, A. Krupski, C. Becker and K. Wandelt, J. Phys. Chem. C, 2009, 113, 13947-13967.

13 F. Coloma, A. Sepúlveda-Escribano, J. L. G. Fierro and F. Rodríguez-Reinoso, Appl. Catal., A, 1996, 136, 231-248.

14 J. Ruiz-Martínez, F. Coloma, A. Sepúlveda-Escribano, J. A. Anderson and F. Rodríguez-Reinoso, Catal. Today, 2008, 133-135, 35-41. 
15 Z. Poltarzewski, S. Galvagno, R. Pietropaolo and P. Staiti, J. Catal., 1986, 102, 190-198.

16 P. Claus, Chem. Ing. Tech., 1995, 67, 1340-1344.

17 W. G. Menezes, L. Altmann, V. Zielasek, K. Thiel and M. Bäumer, J. Catal., 2013, 300, 125-135.

18 E. Merlen, P. Beccat, J. C. Bertolini, P. Delichère, N. Zanier and B. Didillon, J. Catal., 1996, 159, 178-188.

19 A. Iglesias-Juez, A. M. Beale, K. Maaijen, T. C. Weng, P. Glatzel and B. M. Weckhuysen, J. Catal., 2010, 276, 268-279.

20 X. Wang, J. r. Stöver, V. Zielasek, L. Altmann, K. Thiel, K. Al-Shamery, M. Bäumer, H. Borchert, J. r. Parisi and J. Kolny-Olesiak, Langmuir, 2011, 27, 11052-11061.

21 P. Sonström, D. Arndt, X. Wang, V. Zielasek and M. Bäumer, Angew. Chem., Int. Ed., 2011, 50, 3888-3891.

22 L. Altmann, S. Kunz and M. Bäumer, J. Phys. Chem. C, 2014, 118, 8925-8932.

23 Y. Wang, J. Ren, K. Deng, L. Gui and Y. Tang, Chem. Mater., 2000, 12, 1622-1627.

24 X. Wang, L. Altmann, J. Stoever, V. Zielasek, M. Baeumer, K. Al-Shamery, H. Borchert, J. Parisi and J. Kolny-Olesiak, Chem. Mater., 2013, 25, 1400-1407.
25 L. Altmann, X. Wang, J. Stöver, M. Klink, V. Zielasek, K. Thiel, J. Kolny-Olesiak, K. Al-Shamery, H. Borchert, J. Parisi and M. Bäumer, ChemCatChem, 2013, 5, 1803-1810.

26 Z. Paál, A. Wootsch, D. Teschner, K. Lázár, I. E. Sajó, N. Györffy, G. Weinberg, A. Knop-Gericke and R. Schlögl, Appl. Catal., A, 2011, 391, 377-385.

27 R. Rao, A. Dandekar, R. T. K. Baker and M. A. Vannice, J. Catal., 1997, 171, 406-419.

28 M. T. Paffett, S. C. Gebhard, R. G. Windham and B. E. Koel, Surf. Sci., 1989, 223, 449-464.

29 F. Delbecq and P. Sautet, J. Catal., 2003, 220, 115-126.

30 Š. Pick, Surf. Sci., 1999, 436, 220-226.

31 La catalyse en chimie organique, ed. P. Sabatier, Paris, 2nd edn, 1920.

32 J. Haubrich, D. Loffreda, F. Delbecq, Y. Jugnet, P. Sautet, A. Krupski, C. Becker and K. Wandelt, Chem. Phys. Lett., 2006, 433, 188-192.

33 S. Alayoglu, P. Zavalij, B. Eichhorn, Q. Wang, A. I. Frenkel and P. Chupas, ACS Nano, 2009, 3, 3127-3137.

34 N. Homs, J. Llorca, P. R. de la Piscina, F. Rodriguez-Reinoso, A. Sepulveda-Escribano and J. Silvestre-Albero, Phys. Chem. Chem. Phys., 2001, 3, 1782-1788. 